\title{
ON THE LIDSKII-VISHIK-LYUSTERNIK PERTURBATION THEORY FOR EIGENVALUES OF MATRICES WITH ARBITRARY JORDAN STRUCTURE*
}

\author{
JULIO MORO ${ }^{\dagger}$, JAMES V. BURKE ${ }^{\ddagger}$, AND MICHAEL L. OVERTON ${ }^{\S}$ \\ Dedicated to V. B. Lidskii and M. I. Vishik on the respective occasions of their 70 th and \\ 75 th birthdays.
}

\begin{abstract}
Let $A$ be a complex matrix with arbitrary Jordan structure and $\lambda$ an eigenvalue of $A$ whose largest Jordan block has size $n$. We review previous results due to Lidskii [U.S.S.R. Comput. Math. and Math. Phys., 1 (1965), pp. 73-85], showing that the splitting of $\lambda$ under a small perturbation of $A$ of order $\varepsilon$ is, generically, of order $\varepsilon^{1 / n}$. Explicit formulas for the leading coefficients are obtained, involving the perturbation matrix and the eigenvectors of $A$. We also present an alternative proof of Lidskii's main theorem, based on the use of the Newton diagram. This approach clarifies certain difficulties which arise in the nongeneric case and leads, in some situations, to the extension of Lidskii's results. These results suggest a new notion of Hölder condition number for multiple eigenvalues, depending only on the associated left and right eigenvectors, appropriately normalized, not on the Jordan vectors.
\end{abstract}

Key words. perturbation of eigenvalues, perturbation theory for linear operators, stability theory, Newton diagram, Newton envelope, spectral condition number

AMS subject classifications. 15A18, 34D10, 47A55, 65F 15

PII. S0895479895294666

1. Introduction. Given a square complex matrix $A$, it is an important question from both the theoretical and the practical points of view to know how the eigenvalues and eigenvectors change when the elements of $A$ are subjected to small perturbations. The usual formulation of the problem introduces a perturbation parameter $\varepsilon$, belonging to some neighborhood of zero, and writes the perturbed matrix as $A+\varepsilon B$ for an arbitrary matrix $B$. In this situation, it is well known [1, section 9.3.1], [7, section II.1.2] that each eigenvalue or eigenvector of $A+\varepsilon B$ admits an expansion in fractional powers of $\varepsilon$, whose zero-th order term is an eigenvalue or eigenvector of the unperturbed matrix $A$.

In this paper we address the question of determining the first-order term of this expansion or, more precisely, the first nonzero perturbation term. No restriction is imposed on the Jordan structure of $A$, although we assume that this Jordan structure is known from the outset. In section 2 we present two results, due to Lidskii [10], which provide, under certain nondegeneracy conditions, the leading exponents and leading coefficients of both eigenvalue and eigenvector perturbations. The central idea of the

* Received by the editors November 9, 1995; accepted for publication (in revised form) by B. Kågström August 30, 1996.

http://www.siam.org/journals/simax/18-4/29466.html

†Departamento de Matemáticas, Universidad Carlos III, Madrid, Spain (jmoro@ dulcinea.uc3m.es). This work was done while the author was on leave from Universidad Complutense de Madrid, visiting the Courant Institute of Mathematical Sciences, New York University, New York, NY. The work of this author was supported in part by an Ayuda Complutense Postdoctoral en el Extranjero and by CICYT grant TAP94-115.

${ }^{\ddagger}$ Department of Mathematics, University of Washington, Seattle, WA 98195-4350 (burke@ math.washington.edu). The work of this author was supported in part by NSF grant DMS-9303772.

${ }^{\S}$ Computer Science Department, Courant Institute of Mathematical Sciences, New York University, New York, NY (overton@cs.nyu.edu). The work of this author was supported in part by NSF grant CCR-9401136. 
proof is simply to transform the characteristic equation $\operatorname{det}(\omega I-A-\varepsilon B)=0$ into an equivalent one $Q(\mu, z)=0$ through a change of variables

$$
\begin{aligned}
& z=\varepsilon^{1 / n}, \\
& \mu=\frac{\omega-\lambda}{z}
\end{aligned}
$$

for a suitable $n$, where $\lambda$ is an eigenvalue of $A$. An appropriate factorization of $Q(\mu, 0)$ leads to the final result.

A specific example may be helpful to give a better idea of these results: take a $9 \times 9$ Jordan matrix $J$ with a unique zero eigenvalue and four Jordan blocks with respective dimensions $3,3,2$, and 1 . Lidskii's results show that, given a small perturbation $J+\varepsilon B$, every Jordan block of $J$ of dimension $n$ gives rise, generically, to $n$ eigenvalues of the perturbed matrix with leading term $O\left(\varepsilon^{1 / n}\right)$. In this particular case, this amounts to six eigenvalues of order $\varepsilon^{1 / 3}$, two of order $\varepsilon^{1 / 2}$, and one of order $\varepsilon$. As for the coefficients of these leading terms, we will show that they depend exclusively on the elements of $B$ marked with a box in the matrix below:

$$
B=\left[\begin{array}{lllllllll}
* & * & * & * & * & * & * & * & * \\
* & * & * & * & * & * & * & * & * \\
\square & * & * & \square & * & * & \square & * & \square \\
* & * & * & * & * & * & * & * & * \\
* & * & * & * & * & * & * & * & * \\
\square & * & * & \square & * & * & \square & * & \square \\
* & * & * & * & * & * & * & * & * \\
\square & * & * & \square & * & * & \square & * & \square \\
\square & * & * & \square & * & * & \square & * & \square
\end{array}\right] .
$$

More specifically, let $\Phi_{1}$ denote the $2 \times 2$ matrix given by the four boxes at the top left, i.e.,

$$
\Phi_{1}=\left[\begin{array}{ll}
B_{31} & B_{34} \\
B_{61} & B_{64}
\end{array}\right]
$$

and let $\xi_{1}^{1}, \xi_{1}^{2}$ be the eigenvalues of $\Phi_{1}$. Then the perturbed matrix $J+\varepsilon B$ has six eigenvalues with leading terms

$$
\left(\xi_{1}^{k}\right)^{1 / 3} \varepsilon^{1 / 3}, \quad k=1,2,
$$

using all three cube roots of each $\xi_{1}^{k}$. Now, let

$$
\Phi_{2}=\left[\begin{array}{lll}
B_{31} & B_{34} & B_{37} \\
B_{61} & B_{64} & B_{67} \\
\hline B_{81} & B_{84} & B_{87}
\end{array}\right]
$$

and let $\xi_{2}$ denote the Schur complement of $\Phi_{1}$ in $\Phi_{2}$, i.e.,

$$
\xi_{2}=B_{87}-\left[\begin{array}{ll}
B_{81} & B_{84}
\end{array}\right] \Phi_{1}^{-1}\left[\begin{array}{c}
B_{37} \\
B_{67}
\end{array}\right]
$$


Then the two $O\left(\varepsilon^{1 / 2}\right)$ eigenvalues of $J+\varepsilon B$ have leading terms

$$
\left(\xi_{2}\right)^{1 / 2} \varepsilon^{1 / 2} .
$$

Finally, the leading coefficient of the $O(\varepsilon)$ eigenvalue is the Schur complement of $\Phi_{2}$ in the $9 \times 9$ matrix formed by all boxes, i.e.,

$$
\xi_{3}=B_{99}-\left[\begin{array}{lll}
B_{91} & B_{94} & B_{97}
\end{array}\right] \Phi_{2}^{-1}\left[\begin{array}{c}
B_{39} \\
B_{69} \\
B_{89}
\end{array}\right] .
$$

In the most general case when $A$ is not in Jordan form, one must replace the elements of $B$ marked with the boxes by products $y B x$, where $x$ (resp., $y$ ) is a right (resp., left) eigenvector of $A$.

The first results in this direction were obtained by Vishik and Lyusternik [13], motivated by applications to differential operators. Lidskii [10] generalized their results in the finite-dimensional case, obtaining simple explicit formulas for the perturbation coefficients and providing, at the same time, a much more elementary proof (which is essentially the one we present in section 2). The results in both [13] and [10] were later refined by Baumgärtel [1, section 7.4] in the sense of dealing not only with perturbation series for eigenvalues and eigenvectors, but also with the corresponding eigenprojections as functions of $\varepsilon$. Vainberg and Trenogin [12, section 32], on the other hand, offer a fairly thorough account of similar results, obtained for Fredholm operators by applying the techniques of branching theory. Langer and Najman [9] recently generalized Lidskii's results to matrix pencils $M(\lambda)+N(\lambda, \varepsilon)$, using the local Smith normal form of parameter-dependent matrices (Lidskii's results follow from choosing $M \equiv A-\lambda I, N \equiv \varepsilon B$ ). The fundamental results of Lidskii remain, however, almost completely unknown in the Western literature. The only references to [10] appearing in the Science Citation Index are [3] and [9], and both of these continue earlier work [2], [8] in which the authors were unaware of [10]. The main purpose of this paper is, therefore, to bring Lidskii's results to the attention of the broad linear algebra community. See [11] for an application to stability theory for Hamiltonian systems.

Section 2 is devoted to reviewing both the results and the proofs given in [10]. We should stress here that, although Lidskii stated his results as being valid for analytic perturbations, we will see that they hold in fact for a more general class of perturbations, including those of class $C^{1}$ (see Remark 4 in section 2). Lidskii's results, however, depend on certain nondegeneracy assumptions, and no information about the leading exponents or coefficients is available in the degenerate case from the approach taken in section 2. Consider, for instance, the following example taken from Wilkinson [14, section 2.22]: let $A$ be a Jordan matrix with two Jordan blocks of sizes 3 and 2 , which is perturbed only in the positions $(3,4)$ and $(5,1)$, i.e.,

$$
A+\varepsilon B=\left[\begin{array}{ccc|cc}
0 & 1 & & & \\
& 0 & 1 & & \\
& & 0 & \varepsilon & \\
& & & 0 & 1 \\
\varepsilon & & & & 0
\end{array}\right] .
$$

One can easily check that the characteristic polynomial of $A+\varepsilon B$ is $\varepsilon^{2}-\lambda^{5}$. Hence, the eigenvalues of $A+\varepsilon B$ are $O\left(\varepsilon^{2 / 5}\right)$, an order which Lidskii's results are unable to 
predict. We present in section 3 a different approach which will, in particular, reveal the origin of this exponent. Apart from providing an alternative proof of Lidskii's main theorem, the new point of view identifies the difficulties which arise in the degenerate case and, in some situations, leads to extensions of the results in section 2. This alternative approach is much in the spirit of [12] since our main tool is the Newton diagram.

We end by proposing in section 4 a new notion of Hölder condition number for multiple eigenvalues, suggested by Lidskii's results. Although it is closely related to previous Hölder condition numbers in the literature $[4$, p. 156] its main difference is that it depends only on the associated left and right eigenvectors, appropriately normalized, not on the Jordan vectors.

2. Lidskii's perturbation theory. Let $A$ be a complex matrix with Jordan form

$$
\left[\begin{array}{ll}
J & \\
& \widehat{J}
\end{array}\right]=\left[\begin{array}{l}
Q \\
\widehat{Q}
\end{array}\right] A\left[\begin{array}{ll}
P & \widehat{P}
\end{array}\right]
$$

with

$$
\left[\begin{array}{l}
Q \\
\widehat{Q}
\end{array}\right]\left[\begin{array}{ll}
P & \widehat{P}
\end{array}\right]=I,
$$

where $J$ corresponds to a multiple eigenvalue $\lambda$ and $\widehat{J}$ is the part of the Jordan form containing the other eigenvalues of $A$. Let

$$
J=\operatorname{Diag}\left(\Gamma_{1}^{1}, \ldots, \Gamma_{1}^{r_{1}}, \ldots, \Gamma_{q}^{1}, \ldots, \Gamma_{q}^{r_{q}}\right),
$$

where, for $j=1, \ldots, q$,

$$
\Gamma_{j}^{1}=\cdots=\Gamma_{j}^{r_{j}}=\left[\begin{array}{ccccc}
\lambda & 1 & & & \\
& \cdot & \cdot & & \\
& & \cdot & \cdot & \\
& & & \cdot & 1 \\
& & & & \lambda
\end{array}\right]
$$

is a Jordan block of dimension $n_{j}$, repeated $r_{j}$ times, and ordered so that

$$
n_{1}>n_{2}>\cdots>n_{q}
$$

The $n_{j}$ are called the partial multiplicities for $\lambda$. The eigenvalue $\lambda$ is semisimple (nondefective) if $q=n_{1}=1$ and nonderogatory if $q=r_{1}=1$. The algebraic and geometric multiplicities of $\lambda$ are, respectively,

$$
m=\sum_{j=1}^{q} r_{j} n_{j} \quad \text { and } \quad g=\sum_{j=1}^{q} r_{j} .
$$


We further partition

$$
P=\left[\begin{array}{lll|l|l|l|l|l}
P_{1}^{1} & \ldots & P_{1}^{r_{1}} & \ldots & P_{q}^{1} & \ldots & P_{q}^{r_{q}}
\end{array}\right]
$$

conformally with (2.3). The columns of each $P_{j}^{k}$ form a right Jordan chain of $A$ with length $n_{j}$ corresponding to $\lambda$. If we denote by $x_{j}^{k}$ the first column of $P_{j}^{k}$, each $x_{j}^{k}$ is a right eigenvector of $A$ associated with $\lambda$. Analogously, we split

$$
Q=\left[\begin{array}{c}
Q_{1}^{1} \\
\vdots \\
Q_{1}^{r_{1}} \\
\vdots \\
Q_{q}^{1} \\
\vdots \\
Q_{q}^{r_{q}}
\end{array}\right]
$$

also conformally with (2.3). The rows of each $Q_{j}^{k}$ form a left Jordan chain of $A$ of length $n_{j}$ corresponding to $\lambda$. Hence, if we denote by $y_{j}^{k}$ the last (i.e., $n_{j}$ th) row of $Q_{j}^{k}$, each $y_{j}^{k}$ is a left eigenvector corresponding to $\lambda$. With these eigenvectors we build up matrices

$$
Y_{j}=\left[\begin{array}{c}
y_{j}^{1} \\
\vdots \\
y_{j}^{r_{j}}
\end{array}\right], \quad X_{j}=\left[x_{j}^{1}, \ldots, x_{j}^{r_{j}}\right]
$$

for $j=1, \ldots, q$,

$$
W_{s}=\left[\begin{array}{c}
Y_{1} \\
\vdots \\
Y_{s}
\end{array}\right], \quad Z_{s}=\left[X_{1}, \ldots, X_{s}\right]
$$

for $s=1, \ldots, q$, and define square matrices $\Phi_{s}$ and $E_{s}$ of dimension $f_{s}=\sum_{j=1}^{s} r_{j}$ by

$$
\begin{array}{ll}
\Phi_{s}=W_{s} B Z_{s}, & s=1, \ldots, q, \\
E_{1}=I, & E_{s}=\left[\begin{array}{ll}
0 & 0 \\
0 & I
\end{array}\right] \quad \text { for } s=2, \ldots, q,
\end{array}
$$

where the identity block in $E_{s}$ has dimension $r_{s}$. Note that, due to the cumulative definitions of $W_{s}$ and $Z_{s}$, every $\Phi_{s-1}, s=2, \ldots, q$, is the upper left block of $\Phi_{s}$. 
Theorem 2.1 (due to Lidskii [10]). Let $j \in\{1, \ldots, q\}$ be given and assume that, if $j>1, \Phi_{j-1}$ is nonsingular. Then there are $r_{j} n_{j}$ eigenvalues of the perturbed matrix $A+\varepsilon B$ admitting a first-order expansion

$$
\lambda_{j}^{k l}(\varepsilon)=\lambda+\left(\xi_{j}^{k}\right)^{1 / n_{j}} \varepsilon^{1 / n_{j}}+o\left(\varepsilon^{1 / n_{j}}\right)
$$

for $k=1, \ldots, r_{j}, l=1, \ldots, n_{j}$, where

(i) the $\xi_{j}^{k}, k=1, \ldots, r_{j}$, are the roots of equation

$$
\operatorname{det}\left(\Phi_{j}-\xi E_{j}\right)=0
$$

or, equivalently, the eigenvalues of the Schur complement of $\Phi_{j-1}$ in $\Phi_{j}$ (if $j=1$, the $\xi_{1}^{k}$ are just the $r_{1}$ eigenvalues of $\left.\Phi_{1}\right)$,

(ii) the different values $\lambda_{j}^{k l}(\varepsilon)$ for $l=1, \ldots, n_{j}$ are defined by taking the $n_{j}$ distinct $n_{j}$ th roots of $\xi_{j}^{k}$.

If, in addition, the $r_{j}$ solutions $\xi_{j}^{k}$ of (2.5) are all distinct, then the eigenvalues (2.4) can be expanded locally in a power series of the form

$$
\lambda_{j}^{k l}(\varepsilon)=\lambda+\left(\xi_{j}^{k}\right)^{1 / n_{j}} \varepsilon^{1 / n_{j}}+\sum_{s=2}^{\infty} a_{j s}^{k l} \varepsilon^{s / n_{j}}
$$

$k=1, \ldots, r_{j}, l=1, \ldots, n_{j}$.

Remark. Two special cases of Theorem 2.1 are well known. In the case in which $\lambda$ is semisimple, i.e., $q=n_{1}=1$ with multiplicity $r_{1}$, equation (2.4) reduces to

$$
\lambda_{1}^{k 1}(\varepsilon)=\lambda+\xi_{1}^{k} \varepsilon+o(\varepsilon),
$$

where the $\xi_{1}^{k}$ are the eigenvalues of the $r_{1} \times r_{1}$ matrix $Y_{1} B X_{1}$ (cf. [7, section II.2.3]). In the case in which $\lambda$ is nonderogatory, i.e., $q=r_{1}=1$ with multiplicity $n_{1}$, equation (2.4) reduces to

$$
\lambda_{j}^{1 l}(\varepsilon)=\lambda+\left(\xi_{1}^{1}\right)^{1 / n_{1}} \varepsilon^{1 / n_{1}}+o\left(\varepsilon^{1 / n_{1}}\right),
$$

where $\xi_{1}^{1}=y_{1}^{1} B x_{1}^{1}$. These two cases coincide when $\lambda$ is simple.

TheOrem 2.2 (due to Lidskii [10]). Let $\Phi_{s}$ be nonsingular for $s=1, \ldots, q$ and let $j \in\{1, \ldots, q\}$ be such that the $r_{j}$ roots of (2.5) are different. Then the corresponding eigenvalues (2.6) of $A+\varepsilon B$ are simple for $\varepsilon$ small enough and the associated right eigenvectors admit a power series expansion

$$
v_{j}^{k l}(\varepsilon)=u_{j}^{k}+\sum_{s=1}^{\infty} w_{j s}^{k l} \varepsilon^{s / n_{j}},
$$

$k=1, \ldots, r_{j}, l=1, \ldots, n_{j}$, where

$$
u_{j}^{k}=\sum_{p=1}^{f_{j}} c_{j}^{k p} x_{j}^{p}, \quad k=1, \ldots, r_{j}
$$


and the column vector

$$
c_{j}^{k}=\left[\begin{array}{c}
c_{j}^{k 1} \\
\vdots \\
c_{j}^{k f_{j}}
\end{array}\right]
$$

satisfies

$$
\left(\Phi_{j}-\xi_{j}^{k} E_{j}\right) c_{j}^{k}=0 .
$$

Proof of Theorem 2.1. We may suppose, for the sake of simplicity, that $A$ has only one eigenvalue $\lambda$; i.e., $\widehat{J}$ is empty. The general case may be reduced to this one using appropriate Riesz projections (we refer to Lidskii's original paper [10, pp. 83-84] or [1, section 3.9.1] for the details). Thus, we are interested in the roots $\omega$ of the characteristic equation

$$
\operatorname{det} C(\omega, \varepsilon) \equiv \operatorname{det}(\omega I-J-\varepsilon \widetilde{B})=0, \quad \widetilde{B}=P^{-1} B P .
$$

As announced in section 1 , we perform on $C(\omega, \varepsilon)$ the change of variables

$$
\begin{aligned}
& z=\varepsilon^{1 / n_{j}}, \\
& \mu=\frac{\omega-\lambda}{z},
\end{aligned}
$$

where $n_{j}$ is the partial multiplicity corresponding to $j$. This leads to a polynomial equation

$$
\operatorname{det} \mathcal{P}(\mu, z)=\operatorname{det}\left[(\lambda+\mu z) I-J-z^{n_{j}} \widetilde{B}\right]=0
$$

in the new variables, where $\mathcal{P}(\mu, z)=C\left(\lambda+\mu z, z^{n_{j}}\right)$. Since we are mainly concerned with solutions which are close to $z=0$, it will prove convenient to multiply $\mathcal{P}(\mu, z)$ by the following diagonal matrices $L(z)$ and $R(z)$, partitioned conformally with $J$ :

$$
\begin{aligned}
& L(z)=\operatorname{Diag}\left[L_{1}^{1}, \ldots, L_{1}^{r_{1}}, \ldots, L_{q}^{1}, \ldots, L_{q}^{r_{q}}\right] \\
& R(z)=\operatorname{Diag}\left[R_{1}^{1}, \ldots, R_{1}^{r_{1}}, \ldots, R_{q}^{1}, \ldots, R_{q}^{r_{q}}\right]
\end{aligned}
$$

where

$$
\begin{array}{ll}
L_{i}^{1}(z)=\cdots=L_{i}^{r_{i}}(z)=\operatorname{diag}\left[z^{-1}, z^{-2}, \ldots, z^{-n_{i}}\right] & \text { if } i \geq j, \\
L_{i}^{1}(z)=\cdots=L_{i}^{r_{i}}(z)=\operatorname{diag}[\underbrace{1, \ldots, 1}_{n_{i}-n_{j}}, z^{-1}, z^{-2}, \ldots, z^{-n_{j}}] & \text { if } i<j
\end{array}
$$

and

$$
\begin{array}{ll}
R_{i}^{1}(z)=\cdots=R_{i}^{r_{i}}(z)=\operatorname{diag}\left[1, z, z^{2}, \ldots, z^{n_{i}-1}\right] & \text { if } i \geq j, \\
R_{i}^{1}(z)=\cdots=R_{i}^{r_{i}}(z)=\operatorname{diag}[\underbrace{1, \ldots, 1}_{n_{i}-n_{j}}, 1, z, z^{2}, \ldots, z^{n_{j}-1}] & \text { if } i<j
\end{array}
$$


for $i=1, \ldots, q$ (note that $n_{i} \geq n_{j}$ if and only if $i \leq j$ ). We now introduce the matrix $F(\mu, z)=L(z) \mathcal{P}(\mu, z) R(z)$ and define

$$
\mathcal{Q}(\mu, z)=\operatorname{det} F(\mu, z) .
$$

The nonsingularity of both $L(z)$ and $R(z)$ implies that, for any given $z \neq 0$,

$$
\operatorname{det} \mathcal{P}(\mu, z)=0 \Leftrightarrow \mathcal{Q}(\mu, z)=0,
$$

although of course the condition numbers of $L(z), R(z)$ diverge to $\infty$ as $z \rightarrow 0$.

Let us show that $\mathcal{Q}$ is a polynomial in $\mu$ and $z$. For this, we split $F(\mu, z)=$ $G(\mu, z)+H(z)$, where

$$
G(\mu, z)=L(z)[(\lambda+\mu z) I-J] R(z)
$$

is block diagonal and

$$
H(z)=-z^{n_{j}} L(z) \widetilde{B} R(z) .
$$

We write $\Gamma_{s}^{k}=\lambda I+N_{s}, k=1, \ldots, r_{s}$, where

$$
N_{s}=\left[\begin{array}{ccccc}
0 & 1 & & & \\
& \cdot & \cdot & & \\
& & \cdot & \cdot & \\
& & & \cdot & 1 \\
& & & & 0
\end{array}\right]
$$

for $s=1, \ldots, q$, and use two straightforward properties of the matrices $L_{i}^{k}$ and $R_{i}^{k}$, namely, that

$$
\begin{aligned}
& L_{i}^{k}(z) N_{i} R_{i}^{k}(z)=N_{i}, \quad i=1, \ldots, q, \quad k=1, \ldots, r_{i}, \\
& L_{i}^{k}(z) R_{i}^{k}(z)=z^{-1} I \quad \text { whenever } n_{i} \leq n_{j},
\end{aligned}
$$

to check that the diagonal blocks $G_{i}^{k}(\mu, z)=L_{i}^{k}\left(\mu z I-N_{i}\right) R_{i}^{k}$ of $G(\mu, z)$ are

$$
G_{i}^{k}(\mu, z)= \begin{cases}\mu I-N_{i} & \text { if } i \geq j, \\ \operatorname{diag}[\underbrace{\mu z, \ldots, \mu z}_{n_{i}-n_{j}}, \mu, \ldots, \mu]-N_{i} & \text { if } i<j .\end{cases}
$$

Hence, all powers of $z$ in $G$ are nonnegative, and the same applies to $H$ since no negative powers appear in either $z^{n_{j}} L_{i}^{k}$ or $R_{i}^{k}$. This proves our claim that $\mathcal{Q}(\mu, z)$ is a polynomial.

Let us now examine $F(\mu, 0)=G(\mu, 0)+H(0)$. The block diagonal matrix $G(\mu, 0)$ is given by equations (2.10) with $z=0$. To give a careful description of $H(0)$ we need to partition $\widetilde{B}, F$, and $H$ conformally with $J$. We denote by

$$
\widetilde{B}_{j_{1} j_{2}}^{k_{1} k_{2}}, \quad j_{i}=1, \ldots, q, \quad k_{i}=1, \ldots, r_{j_{i}}, \quad i=1,2
$$


the $n_{j_{1}} \times n_{j_{2}}$ block of $\widetilde{B}$ lying on the same rows as $\Gamma_{j_{1}}^{k_{1}}$ and on the same columns as $\Gamma_{j_{2}}^{k_{2}}$ (the corresponding blocks of $H$ and $F$ are defined likewise). Following this notation, we have

$$
H_{j_{1} j_{2}}^{k_{1} k_{2}}(z)=-z^{n_{j}} L_{j_{1}}^{k_{1}}(z) \widetilde{B}_{j_{1} j_{2}}^{k_{1} k_{2}} R_{j_{2}}^{k_{2}}(z),
$$

which implies, in the first place, that

$$
H_{j_{1} j_{2}}^{k_{1} k_{2}}(0)=0 \quad \text { if } n_{j_{1}}<n_{j}
$$

due to the vanishing of $z^{n_{j}} L_{j_{1}}^{k_{1}}$ at $z=0$. On the other hand, if $j_{1} \leq j$ and $j_{2} \leq j$, an elementary calculation shows that

$$
H_{j_{1} j_{2}}^{k_{1} k_{2}}(0)=\left[\begin{array}{ccccccc}
0 & 0 & \cdots & 0 & 0 & \cdots & 0 \\
\cdot & \cdot & \cdots & \cdot & \cdot & \cdots & \cdot \\
0 & 0 & \cdots & 0 & 0 & \cdots & 0 \\
-\beta_{j_{1} j_{2}}^{k_{1} k_{2}} & * & \cdots & * & 0 & \cdots & 0
\end{array}\right],
$$

where the $n_{j_{2}}-n_{j}$ elements marked with an asterisk are irrelevant to our argument and $\beta_{j_{1} j_{2}}^{k_{1} k_{2}}$ is the element in the lower left corner of the block $\widetilde{B}_{j_{1} j_{2}}^{k_{1} k_{2}}$. The same structure (2.12) applies to the case $j_{1} \leq j, j_{2}>j$, but with zeros instead of asterisks. The main point of (2.12) is that every $\beta_{j_{1} j_{2}}^{k_{1} k_{2}}=y_{j_{1}}^{k_{1}} B x_{j_{2}}^{k_{2}}$ is an element of $\Phi_{s}=Y_{s} B X_{s}$ for $s=\max \left\{j_{1}, j_{2}\right\}$. In other words, we may find all the elements of the matrix $\Phi_{j}$ by looking at the lower left corners of the blocks (2.12) for $j_{1}, j_{2} \leq j$ (or, equivalently, for $\left.n_{j_{1}}, n_{j_{2}} \geq n_{j}\right)$.

Before our final examination of $\mathcal{Q}(\mu, 0)$, let us briefly turn to the diagonal blocks of $F(\mu, 0)$ of size $n_{j}$, i.e.,

$$
F_{j j}^{k k}(\mu, 0)=\left[\begin{array}{ccccc}
\mu & -1 & 0 & \cdots & 0 \\
0 & \mu & -1 & \cdots & 0 \\
\cdot & \cdot & \cdot & \cdots & \cdot \\
-\beta_{j j}^{k k} & 0 & 0 & \cdots & \mu
\end{array}\right]
$$

The determinant of this block does not change if we add to its first column the products of its second column by $\mu$, of its third column by $\mu^{2}, \ldots$, and of its last column by $\mu^{n_{j}-1}$. Neither does the whole determinant $\mathcal{Q}(\mu, 0)$ if we perform identical operations on the same columns of the whole matrix $F(\mu, 0)$ since, according to (2.11) and (2.12), the $n_{j}-1$ columns of $F$ which are being multiplied by powers of $\mu$ have no nonzero elements outside $F_{j j}^{k k}$. This amounts to replacing every block $F_{j j}^{k k}$ with the block

$$
\left[\begin{array}{ccccc}
0 & -1 & 0 & \cdots & 0 \\
0 & \mu & -1 & \cdots & 0 \\
\cdot & \cdot & \cdot & \cdots & \cdot \\
-\beta_{j j}^{k k}+\mu^{n_{j}} & 0 & 0 & \cdots & \mu
\end{array}\right] .
$$

Let us now prove that the determinant $\mathcal{Q}(\mu, 0)$ of the matrix we finally obtain can be written in the form 


$$
\mathcal{Q}(\mu, 0)= \pm \mu^{\alpha} \operatorname{det}\left(\Phi_{j}-\mu^{n_{j}} E_{j}\right)
$$

for a suitable $\alpha \geq 0$. Although elementary, the proof is quite messy in the general case, so we will instead illustrate the strategy on a specific example. Take, for instance, the case $q=3, j=2, n_{1}=4, n_{2}=3, n_{3}=2, r_{1}=1, r_{2}=2, r_{3}=1$; i.e., $\mathcal{Q}(\mu, 0)$ is the determinant of the $12 \times 12$ matrix

$$
\left[\begin{array}{cccccccccccc}
0 & -1 & 0 & 0 & 0 & 0 & 0 & 0 & 0 & 0 & 0 & 0 \\
0 & \mu & -1 & 0 & 0 & 0 & 0 & 0 & 0 & 0 & 0 & 0 \\
0 & 0 & \mu & -1 & 0 & 0 & 0 & 0 & 0 & 0 & 0 & 0 \\
-\beta_{11}^{11} & * & 0 & \mu & -\beta_{12}^{11} & * & 0 & -\beta_{12}^{12} & 0 & 0 & -\beta_{13}^{11} & 0 \\
0 & 0 & 0 & 0 & 0 & -1 & 0 & 0 & 0 & 0 & 0 & 0 \\
0 & 0 & 0 & 0 & 0 & \mu & -1 & 0 & 0 & 0 & 0 & 0 \\
-\beta_{21}^{11} & 0 & 0 & \mu & -\beta_{22}^{11}+\mu^{3} & 0 & \mu & -\beta_{22}^{12} & 0 & 0 & -\beta_{23}^{11} & 0 \\
0 & 0 & 0 & 0 & 0 & 0 & 0 & 0 & -1 & 0 & 0 & 0 \\
0 & 0 & 0 & 0 & 0 & 0 & 0 & 0 & \mu & -1 & 0 & 0 \\
-\beta_{21}^{21} & 0 & 0 & \mu & -\beta_{22}^{21} & 0 & 0 & -\beta_{22}^{22}+\mu^{3} & 0 & \mu & -\beta_{23}^{21} & 0 \\
0 & 0 & 0 & 0 & 0 & 0 & 0 & 0 & 0 & 0 & \mu & -1 \\
0 & 0 & 0 & 0 & 0 & 0 & 0 & 0 & 0 & 0 & 0 & \mu
\end{array}\right] .
$$

There are four rows in this matrix (first, fifth, eighth, and twelfth) containing one single nonzero element. In calculating the determinant of the matrix we may, therefore, remove the rows and columns corresponding to these four elements. This leaves an $8 \times 8$ matrix $M_{1}(\mu)$ such that $\mathcal{Q}(\mu, 0)=\mu$ det $M_{1}(\mu)$. Because of the previous deletions, there are again four rows in $M_{1}$ with one single nonzero element. Eliminating the appropriate rows and columns of $M_{1}$, we get a $4 \times 4$ matrix $M_{2}$ and, finally, deleting one row and one column of $M_{2}$, a $3 \times 3$ matrix

$$
M_{3}=\left[\begin{array}{ccc}
-\beta_{11}^{11} & -\beta_{12}^{11} & -\beta_{12}^{12} \\
-\beta_{21}^{11} & -\beta_{22}^{11}+\mu^{3} & -\beta_{22}^{12} \\
-\beta_{21}^{21} & -\beta_{22}^{21} & -\beta_{22}^{22}+\mu^{3}
\end{array}\right]
$$

such that $\mathcal{Q}(\mu, 0)=\mu^{2} \operatorname{det} M_{3}(\mu)$. But we know from (2.12) that the $\beta_{j_{1} j_{2}}^{k_{1} k_{2}}$ are just the elements of $\Phi_{2}$. Hence, $M_{3}(\mu)=-\Phi_{2}+\mu^{3} E_{2}$ and we obtain (2.13) with $\alpha=2$. The same procedure goes through to the general case, exploiting in much the same way our knowledge of the block structure of the modified matrix $F(\mu, 0)$.

Once we have $\mathcal{Q}(\mu, 0)$ factorized as in (2.13), we note that its second factor $\operatorname{det}\left(\Phi_{j}-\mu^{n_{j}} E_{j}\right)$ is a polynomial of degree $r_{j}$ in $\mu^{n_{j}}$. This is trivial if $j=1$ and a consequence of the nonsingularity of $\Phi_{j-1}$ if $j>1$. We now take $\mathcal{Q}(\mu, z)$ as a polynomial in $\mu$ whose coefficients are continuous $z$-dependent functions. The continuous dependence of the roots of $\mathcal{Q}$ upon its coefficients guarantees the existence of exactly $r_{j} n_{j}$ continuous functions

$$
\mu_{j}^{k l}(z)=\left(\xi_{j}^{k}\right)^{1 / n_{j}}+o(1), \quad k=1, \ldots, r_{j}, \quad l=1, \ldots, n_{j},
$$

describing all solutions of $\mathcal{Q}(\mu(z), z)=0$ for $z$ small enough (recall that some roots $\xi_{j}^{k}$ of (2.5) might be zero if $\Phi_{j}$ is singular). Expansion (2.4) is obtained by returning to the original variables $(\lambda, \varepsilon)$. 
Finally, if all $r_{j}$ roots $\xi_{j}^{k}$ of equation (2.5) are known to be distinct, we may apply the implicit function theorem to $(2.13)$ to show that the $\mu_{j}^{k l}(z)$ are in fact analytic functions of $z$, thus giving rise to the power series (2.6).

Proof of Theorem 2.2. In the conditions of Theorem 2.2 it is clear that, for $\varepsilon$ small enough, no eigenvalue (2.6) corresponding to $j$ can possibly coincide with any of the eigenvalues (2.4) corresponding to Jordan blocks of different dimensions.

Furthermore, given one of these simple eigenvalues $\lambda_{j}^{k l}(\varepsilon)$, a right eigenvector associated with it may be constructed by taking as its components the $m$ cofactors of the elements of a row of $A+\varepsilon B-\lambda_{j}^{k l} I$. This implies the analyticity of the eigenvector since the elements of this latter matrix are analytic functions of $\varepsilon$ and the cofactors are simply sums of products of these elements (we recall that the eigenvector is unique up to constant multiples due to the simplicity of the eigenvalue).

Finally, let $e_{j}^{k l}(z)$ be a vector in the null space of $F_{j}^{k l}(z)=F\left(\mu_{j}^{k l}(z), z\right)$, where $\mu_{j}^{k l}(z)=\left(\lambda_{j}^{k l}(z)-\lambda\right) / z$. Dropping for simplicity both sub- and superscripts, we have

$$
F(z) e(z)=L(z) \mathcal{P}(\mu(z), z) R(z) e(z)=0,
$$

which, due to the nonsingularity of $L(z)$ for $z \neq 0$, shows that $R(z) e(z)$ is a right eigenvector of $J+z^{n_{j}} \widetilde{B}$ associated with $\lambda_{j}^{k l}$. Hence, the first term $R(0) e(0)$ of its $\varepsilon$ expansion must be, up to a constant, equal to the zero-th order term $u_{j}^{k}$ of expansion (2.7). Equation (2.8) is finally obtained by applying to the linear system $F(0) e=0$ the same ideas we used to simplify $\mathcal{Q}(\mu, 0)$ in the proof of Theorem 2.1.

Remarks.

1. A proof of the existence of power series expansions (2.6) and (2.7) under the conditions of Theorem 2.2 goes back to Vishik and Lyusternik [13, Theorem 6, Appendix I]. Their approach, however, is radically different from Lidskii's, since they impose both expansions (2.6) and (2.7) as formal series at the outset, recursively find all coefficients, and finally prove the convergence of the series on some nontrivial interval around $\varepsilon=0$. In their setting, the assumption that all Schur complements have nonzero distinct eigenvalues arises as a solvability condition on the system of infinitely many equations determining the coefficients of the series. Lidskii's approach in [10], on the other hand, concentrates only on the leading term, regardless of the rest of the expansion. This allows him to get more general results, avoiding at the same time the issue of convergence of the series: in those cases in which a power series expansion is obtained, its convergence is a consequence of the function theoretical results invoked in the proof.

2. The computation of Schur complements is equivalent to (and may be replaced by) choosing the eigenvector matrices $X_{j}$ and $Y_{j}$ in a special way. Suppose, for instance, that $j=2$ and $\Phi_{1}=Y_{1} B X_{1}$ is nonsingular. A straightforward calculation shows that the columns of

$$
\tilde{X}_{2}=X_{2}-X_{1} \Phi_{1}^{-1} Y_{1} B X_{2}
$$

and the rows of

$$
\widetilde{Y}_{2}=Y_{2}-Y_{2} B X_{1} \Phi_{1}^{-1} Y_{1}
$$

are, respectively, right and left eigenvectors of $A$, corresponding to Jordan chains of the same length as the eigenvectors given by the rows and columns of $X_{2}$ and $Y_{2}$. 
Furthermore, we have $Y_{1} B \widetilde{X}_{2}=0$ and $\widetilde{Y}_{2} B X_{1}=0$. Hence, it suffices to define

$$
\widetilde{W}_{2}=\left[\begin{array}{c}
Y_{1} \\
\widetilde{Y}_{2}
\end{array}\right], \quad \widetilde{Z}_{2}=\left[X_{1}, \widetilde{X}_{2}\right], \quad \text { and } \quad \widetilde{\Phi}_{2}=\widetilde{W}_{2} B \widetilde{Z}_{2}
$$

to obtain a block diagonal matrix $\widetilde{\Phi}_{2}$ whose lower right block $\widetilde{Y}_{2} B \widetilde{X}_{2}$ is precisely the Schur complement of $\Phi_{1}$ in the old matrix $\Phi_{2}$. The replacement of $X_{3}$ and $Y_{3}$ by suitable matrices leads to the block diagonalization of $\Phi_{3}$, provided that $\widetilde{\Phi}_{2}$ is nonsingular. It should be noted that, although only one of the two matrices (2.14) or (2.15) is required to reproduce the Schur complement for $j=2$ (by block triangularizing $\Phi_{2}$ ), both of them are needed to continue to the following step $j=3$.

3. Both matrices $\Phi_{j-1}$ and $\Phi_{j}$ must be nonsingular to obtain the leading nonzero terms in all expansions (2.4). That is probably why Lidskii's original statement of Theorem 2.1 imposed nonsingularity of both $\Phi_{j-1}$ and $\Phi_{j}$. Nevertheless, as we have seen in the proof of the theorem, only $\Phi_{j-1}$ need be nonsingular for the eigenvalue expansions (2.4) to hold: suppose that $\Phi_{j-1}$ is nonsingular and $\Phi_{j}$ is singular. Then we have

$$
\operatorname{det}\left(\Phi_{j}-\xi E_{j}\right)=\xi^{\beta} q(\xi), \quad q(0) \neq 0,
$$

for a certain $\beta>0$; i.e., (2.5) has $\beta$ zero and $r_{j}-\beta$ nonzero solutions. Hence, $\left(r_{j}-\beta\right) n_{j}$ expansions (2.4) have a nonzero first-order term, while all we can say about the remaining $\beta n_{j}$ eigenvalues is that they are of the form $\lambda_{j}^{k}(\varepsilon)=\lambda+o\left(\varepsilon^{1 / n_{j}}\right)$. This strongly suggests the possibility of interaction with eigenvalues associated with Jordan blocks of size less than $n_{j}$. These interactions will become much clearer in the next section with the use of the Newton diagram.

4. An important advantage of Lidskii's proof technique is that it does not require the analyticity of the perturbation. Consequently, Lidskii's approach can be used to investigate the variational behavior of eigenvalues under very weak differentiability hypotheses. For example, Theorem 2.1 remains valid for perturbations of class $C^{1}$. More generally, one can even obtain one-sided or directional versions of Theorem 2.1. For example, if $A: \mathbb{R} \rightarrow \mathbb{C}^{n \times n}$ is continuous at the origin with

$$
A(\varepsilon)=A+\varepsilon B+o(\varepsilon) \text { for } \varepsilon>0,
$$

then the expansion (2.4) in Theorem 2.1 remains valid for $\varepsilon \geq 0$. In fact, the same proof holds with minor changes. First observe that the continuity of the Riesz projections depends only on the continuity of the perturbation [7, Theorem 5.1]. Next, using a bar to denote matrices arising in this new setting, we find that

$$
\operatorname{det} C(\omega, \varepsilon) \equiv \operatorname{det}(\omega I-A-\varepsilon B-o(\varepsilon))=\operatorname{det} C(\omega, \varepsilon)+o(\varepsilon) \quad \text { for } \varepsilon \geq 0,
$$

where $C(\omega, \varepsilon)$ is given by (2.9). After changing to variables $\mu, z$, we have

$$
\operatorname{det} \mathcal{P}(\mu, z)=\operatorname{det} \mathcal{P}(\mu, z)+o\left(z^{n_{j}}\right),
$$

so, multiplying by $L(z)$ and $R(z)$, we obtain

$$
F(\mu, z)=L(z) \mathcal{P}(\mu, z) R(z)=F(\mu, z)+S(z)
$$

for $S(z)=L(z) M R(z)$, where $M$ is $o\left(z^{n_{j}}\right)$. Now, recall that no negative power of $z$ in $L(z)$ has absolute value larger than $n_{j}$. This means that $S(z)=o(1)$, so

$$
\mathcal{Q}(\mu, 0)=\operatorname{det} F(\mu, 0)=\mathcal{Q}(\mu, 0)
$$


and the factorization (2.13) still holds. Finally, although in this case $\mathcal{Q}(\mu, z)$ is no longer a polynomial in both variables $\mu$ and $z$, it is a polynomial in $\mu$, whose coefficients are continuous functions of $z$. Thus, we may still guarantee that the roots of $\mathcal{Q}(\mu, z)$ depend continuously on $z$ to conclude the proof.

Example. We consider the simplest case of a matrix having an eigenvalue which is neither semisimple nor nonderogatory: let $A$ be a $3 \times 3$ matrix with a triple eigenvalue $\lambda$ of geometric multiplicity two (in our notation, $q=2, n_{1}=2, n_{2}=1, r_{1}=r_{2}=1$ ). Dropping the superscripts, we denote the two left eigenvectors by $y_{1}, y_{2}$ and the two right eigenvectors by $x_{1}, x_{2}$. We find that $\Phi_{1}=\beta_{11}=y_{1} B x_{1}$ and

$$
\Phi_{2}=\left[\begin{array}{ll}
\beta_{11} & \beta_{12} \\
\beta_{21} & \beta_{22}
\end{array}\right], \quad \beta_{i j}=y_{i} B x_{j}, \quad i, j=1,2 .
$$

We have two eigenvalues

$$
\lambda_{1}^{l}(\varepsilon)=\lambda \pm \sqrt{ } \beta_{11} \varepsilon^{1 / 2}+o\left(\varepsilon^{1 / 2}\right), \quad l=1,2 .
$$

Furthermore, if both $\Phi_{1}$ and det $\Phi_{2}$ are different from zero, the third eigenvalue is $\lambda+\xi_{2} \varepsilon+o(\varepsilon)$, where $\xi_{2}=\left(\operatorname{det} \Phi_{2}\right) / \Phi_{1}$ is the solution of

$$
\operatorname{det}\left(\Phi_{2}-\xi E_{2}\right)=\operatorname{det} \Phi_{2}-\Phi_{1} \xi=0
$$

Note that, if $\Phi_{1}$ is zero, we know only that two of the eigenvalues are $o\left(\varepsilon^{1 / 2}\right)$ perturbations of $\lambda$, without any further indication of their asymptotic order.

Note that even in this simple example it is unclear which leading powers of $\varepsilon$ are to be expected when some $\Phi_{j}$ is singular. Lidskii [10] gives an example where all three eigenvalues above are perturbed by order $\varepsilon^{2 / 3}$, and we have seen in (1.1) a similar example of a $5 \times 5$ matrix $A$ with Jordan blocks of sizes 3 and 2, whose perturbed eigenvalues are of order $\varepsilon^{2 / 5}$ for a conveniently chosen perturbation. None of these leading exponents can be explained, in principle, by any of the above results. It seems that the information that $\mathcal{Q}(\mu, 0)$ provides in the case of singular $\Phi_{j}$ is helpful only in predicting which powers of $\varepsilon$ cannot appear in the eigenvalue expansions. In the following section we present an alternative approach that will improve our understanding of Theorem 2.1, giving us a global picture of what happens in the degenerate case when some $\Phi_{j}$ is singular.

3. Application of Newton's diagram. In this section the symbol $\lambda$ is used as a parameter, not as a fixed value as earlier. We consider a complex polynomial equation

$$
P(\lambda, \varepsilon)=\lambda^{m}+\alpha_{1}(\varepsilon) \lambda^{m-1}+\cdots+\alpha_{m-1}(\varepsilon) \lambda+\alpha_{m}(\varepsilon)=0
$$

in $\lambda$, with analytic coefficients

$$
\alpha_{k}(\varepsilon)=\hat{\alpha}_{k} \varepsilon^{a_{k}}+\cdots, \quad k=1, \ldots, m,
$$

where $a_{k}$ is the leading exponent and $\hat{\alpha}_{k}$ the leading coefficient of $\alpha_{k}(\varepsilon)$ (i.e., $\hat{\alpha}_{k} \neq 0$ and no term of order lower than $a_{k}$ appears in the expansion of $\left.\alpha_{k}(\varepsilon)\right)$. It is well 
known [1], [7] that the roots of (3.1) are given by expansions in fractional powers of $\varepsilon$. The leading exponents of these expansions can be easily found through the following elementary geometrical construction: we plot the values $a_{k}$ versus $k$ for $k=1, \ldots, m$ together with the point $(0,0)$ corresponding to $\lambda^{m}$ (if $\alpha_{j}(\varepsilon) \equiv 0$, the corresponding point is disregarded). Then we draw the segments on the lower boundary of the convex hull of the plotted points. These segments constitute the so-called Newton diagram associated with $P(\lambda, \varepsilon)$ (see Fig. 3.1 for two specific examples). One can prove $[1$,

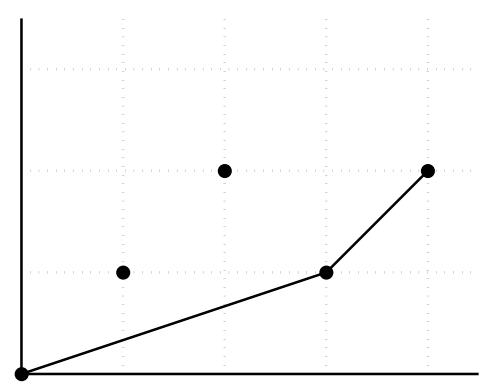

(a)

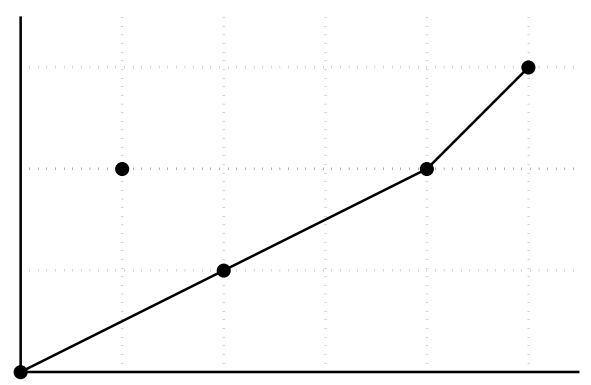

(b)

FIG. 3.1. Newton diagrams associated with the polynomials: (a) $\lambda^{4}+\left(2 \varepsilon-\varepsilon^{2}\right) \lambda^{3}+\varepsilon^{2} \lambda^{2}+(\varepsilon-$ $\left.\varepsilon^{3}\right) \lambda+\varepsilon^{2}$, (b) $\lambda^{5}-\varepsilon^{2} \lambda^{4}+\left(\varepsilon-3 \varepsilon^{2}\right) \lambda^{3}+\varepsilon^{2} \lambda-\varepsilon^{3}$.

Appendix A7], [2], [12] that the slopes of the different segments of the Newton diagram are precisely the leading powers of the $\varepsilon$-expansions of the roots $\lambda=\lambda(\varepsilon)$ of (3.1). The number of roots corresponding to each slope equals the length of the projection on the horizontal axis of the segment with that particular slope. The underlying idea is to substitute an Ansatz

$$
\lambda(\varepsilon)=\mu \varepsilon^{\beta}+\cdots
$$

into (3.1), with $\mu, \beta$ to be determined. Every point $\left(k, a_{k}\right)$ plotted in the diagram produces an $\varepsilon^{a_{k}+(m-k) \beta}$ term. If $\lambda(\varepsilon)$ is a root of (3.1), all the terms we obtain from this substitution must cancel each other. Hence, at least two terms of the lowest order in $\varepsilon$ must be present, and this lowest order is clearly to be found among the exponents $\left\{a_{1}+(m-1) \beta, a_{2}+(m-2) \beta, \ldots, a_{m}\right\}$. Consider the segment $S$ of the Newton diagram with the smallest slope $s$ and choose $\beta=s$ in (3.2). All points $\left(k, a_{k}\right)$ lying on $S$ give rise to terms with the same exponent since $a_{k}+(m-k) s$ is constant on $S$. The fact that no point $\left(k, a_{k}\right)$ lies below $S$ implies that no other term of the expansion can be of a lower order in $\varepsilon$. Hence, the leading coefficients $\mu$ are determined as the solutions of

$$
\sum_{\left(k, a_{k}\right) \in S} \mu^{m-k} \hat{\alpha}_{k}=0
$$

We get the leading terms of the remaining roots of (3.1) by repeating the same argument for the increasingly larger slopes appearing in the Newton diagram.

Returning to the eigenvalue problem, our main goal in this section is to establish the relationship between the quantities $\operatorname{det} \Phi_{j}, j=1, \ldots, q$, and the Newton diagram associated with the characteristic polynomial of $A+\varepsilon B$. We recall that $A$ is a matrix 
with only one eigenvalue (previously denoted by $\lambda$ ) of multiplicity $m$, with partial multiplicities $n_{j}$, each repeated $r_{j}$ times for $j=1, \ldots, q$. With no loss of generality, we may assume that this eigenvalue is zero. In this case, the characteristic polynomial $p(\lambda, \varepsilon)=\operatorname{det}(\lambda I-A-\varepsilon B)=\operatorname{det}(\lambda I-J-\varepsilon \tilde{B})$ can be written in the form (3.1), with $m=\sum_{1}^{q} r_{j} n_{j}$ (recall that $\lambda$ is no longer an eigenvalue of $A$, but the unknown in the characteristic polynomial). To draw the Newton diagram associated with $p(\lambda, \varepsilon)$, we must know the exponents $a_{k}$ for $k=1, \ldots, m$. This is quite easy if the eigenvalue is semisimple, since the Jordan form $J$ of $A$ is zero and each $\alpha_{k}(\varepsilon)$ equals $\varepsilon^{m-k}$ multiplied by a certain sum of minors of $\tilde{B}$ of dimension $m-k$. In this case, the Newton diagram is formed by one single segment of slope $s=1$. If the eigenvalue is not semisimple, some nontrivial Jordan block appears in $J$, which means that, apart from the $O\left(\varepsilon^{m-k}\right)$ terms, each $\alpha_{k}(\varepsilon)$ will typically contain terms of lower order generated by the ones appearing above the diagonal of $J$. This clearly shows that the effect of nontrivial Jordan blocks is to introduce in the Newton diagram line segments with slopes less than 1 , with the smallest possible slope corresponding to the case of a nonderogatory eigenvalue (one single segment of slope $1 / \mathrm{m}$ ) and the largest possible one to the semisimple case. All possible Newton diagrams for the given multiplicity $m$ lie between these two extremal segments.

We must now carefully determine which points $\left(k, a_{k}\right)$ may appear on the Newton diagram for a particular Jordan structure. To this purpose, it will be useful to find the lowest possible diagram compatible with the given Jordan structure. To do this, we fix every exponent $l$ of $\varepsilon$ and find the largest possible $k=k(l)$ such that there exists a perturbation matrix $B$ for which $a_{k(l)}=l$. This amounts to fixing a height $l$ on the vertical axis of the Newton diagram and determining the rightmost possible point $(k(l), l)$ in the diagram. The following theorem gives us the values $k(l)$ for the exponents $l=1, \ldots, f_{q}$ which are relevant to our argument (we recall that $f_{j}=$ $r_{1}+\cdots+r_{j}$ ) and, more importantly, also provides some coefficients of the characteristic polynomial which are crucial to determine the Newton diagram.

THEOREM 3.1. For every $l, l=1, \ldots, f_{q}$, the corresponding $k(l)$ is equal to the sum of the dimensions of the $l$ largest Jordan blocks of $J$. More precisely, write $f_{0}=0$ and suppose $l=f_{j-1}+\rho$ for some $j=1, \ldots, q$ and $0<\rho \leq r_{j}$. Then

$$
k(l)=r_{1} n_{1}+\cdots+r_{j-1} n_{j-1}+\rho n_{j}
$$

and the coefficient of $\varepsilon^{l}$ in $\alpha_{k(l)}$ is equal to $(-1)^{l}$ multiplied by the sum of all principal minors of $\Phi_{j}$ corresponding to submatrices of dimension $l$ that contain the upper left block $\Phi_{j-1}$ of $\Phi_{j}$ (if $j=1$, all principal minors of dimension $l$ are to be considered). If, in particular, $l=f_{j}$ for some $j \in\{1, \ldots, q\}$, then the coefficient of $\varepsilon^{f_{j}}$ in $\alpha_{k\left(f_{j}\right)}$ is $(-1)^{f_{j}} \operatorname{det} \Phi_{j}$.

Proof. The characteristic polynomial of $A+\varepsilon B$ is a linear combination with coefficients \pm 1 of all possible products of $m$ elements of the matrix $\lambda I-J-\varepsilon \widetilde{B}$, with the restriction that no two factors can be on the same row or the same column.

It is clear that the only way to obtain a product of order $\varepsilon^{l}$ is to choose exactly $m-l$ factors containing $\varepsilon$-independent terms (i.e., "lambdas" or "minus ones"). Furthermore, we should try to include as few lambdas as possible among these factors, since we are looking for the smallest possible power of $\lambda$. However, we are not free to make whatever choices we want. Due to the special position of the $\varepsilon$-independent terms, every time we choose a minus one we are, at the same time, excluding from the product those lambdas which lie on the same row or the same column. Let us 
examine the restrictions. Suppose an admissible choice (i.e., a choice producing a term of order $\varepsilon^{l}$ ) contains $\beta$ minus ones. These $\beta$ choices remove a certain number of lambdas, which depends on the number of Jordan blocks these $\beta$ minus ones are sampled from. This is due to the fact that the first minus one we choose from a particular block excludes two lambdas, while any further minus one from the same block removes only one. Suppose the $\beta$ minus ones were taken from $\gamma$ different blocks. These $\beta$ choices exclude $\beta+\gamma$ lambdas, which, together with the $m-l-\beta$ lambdas which were actually chosen in the product, cannot exceed the total number $m$ of available lambdas. We conclude that $\gamma \leq l$; i.e., we are allowed to sample minus ones from at most $l$ Jordan blocks. Hence, to produce the lowest possible power of $\lambda$ we must exhaust all minus ones from the $l$ largest possible Jordan blocks of $J$, and only then complete with lambdas until we have the $m-l$ factors.

Suppose, in the first place, that $l=f_{j}$ for some $j=1, \ldots, q$. Then there is only one way of choosing these $m-l$ factors: we must choose the $\beta=r_{1}\left(n_{1}-1\right)+$ $\cdots+r_{j}\left(n_{j}-1\right)$ minus ones from the $l$ largest Jordan blocks, plus the $m-l-\beta=$ $r_{j+1} n_{j+1}+\cdots+r_{q} n_{q}$ diagonal lambdas from the remaining Jordan blocks. Thus, we get $k(l)=l+\beta=r_{1} n_{1}+\cdots+r_{j} n_{j}$. Note that if we delete from $\lambda I-J-\varepsilon \tilde{B}$ the rows and columns corresponding to these $m-l$ elements, the $f_{j} \times f_{j}$ remaining matrix is precisely $-\varepsilon \Phi_{j}$, which proves our claim.

The same argument is valid in the case $\rho<r_{j+1}$, although in this case there is more than one way of building up the products: each one corresponds to a different choice of $\rho$ blocks among the $r_{j+1}$ Jordan blocks of dimension $n_{j+1}$, generating a different principal minor of $\Phi_{j}$ to be included in the sum.

Let us now introduce the following definition.

Definition 3.2. Denote $\mathcal{P}_{j} \equiv\left(k\left(f_{j}\right), f_{j}\right)$, and let $S_{j}$ be the segment of slope $1 / n_{j}$ connecting $\mathcal{P}_{j-1}$ with $\mathcal{P}_{j}$ for $j=1, \ldots, q$. We define the Newton envelope corresponding to the Jordan structure of $J$ as the diagram obtained by successively joining the segments $S_{1}, S_{2}, \ldots, S_{q}$.

As a first consequence of Theorem 3.1, note that all points $(k(l), l)$ for $l$ between $f_{j-1}$ and $f_{j}$ lie along the corresponding segment $S_{j}$. Hence, the Newton envelope is indeed the lowest Newton diagram we were looking for. This is not, however, its most interesting feature. Keep in mind that, given a particular $B$, only those points $(k(l), l)$ from the envelope such that $a_{k(l)}=l$ will actually be plotted in the Newton diagram. This means, in particular, that a corner point $\mathcal{P}_{j}$ of the Newton envelope appears on the Newton diagram only if the perturbation $B$ is such that the corresponding coefficient $\pm \operatorname{det} \Phi_{j}$ is nonzero. In other words, the Newton envelope displays the generic behavior of the eigenvalues of $A$ under perturbation, in the sense that it coincides with the Newton diagram in all situations except in those nongeneric cases in which the perturbation $B$ causes one of the $\Phi_{j}$ to be singular.

Theorem 3.1 largely explains the importance of the $\operatorname{det} \Phi_{j}$ in obtaining the exponents $1 / n_{j}$ in the eigenvalue expansions. Furthermore, it clears the way for an independent proof of Lidskii's Theorem 2.1.

Proof of Theorem 2.1. Let us suppose first that $j \in\{2, \ldots, q\}$ is such that $\Phi_{j-1}$ and $\Phi_{j}$ are nonsingular (the case $j=1$ is completely analogous). Then both $\mathcal{P}_{j-1}$ and $\mathcal{P}_{j}$ are among the points plotted to construct the Newton diagram and $S_{j}$ is one of the segments in the diagram (no point $\left(k, a_{k}\right)$ can lie below $S_{j}$ ). Thus, we obtain the leading exponent of expansion (2.4). We also get the leading coefficient by carefully examining, for the segment $S_{j}$, equation (3.3). We first note that the only candidates $\left(k, a_{k}\right)$ to lie on $S_{j}$ are the intermediate points $\mathcal{Q}_{t} \equiv\left(k\left(f_{j}-t\right), f_{j}-t\right), t=$ 
$1, \ldots, r_{j}-1$. The fact that the $\mathcal{Q}_{t}$ are separated from each other by a distance $n_{j}$ on the horizontal axis implies that equation (3.3) depends on $\mu$ only through $\mu^{n_{j}}$. More precisely, let $T$ be the set of values $t \in\left\{1, \ldots, r_{j-1}\right\}$ such that $\mathcal{Q}_{t}$ appears in the Newton diagram. Then

$$
\sum_{\left(k, a_{k}\right) \in S_{j}} \mu^{m-k} \hat{\alpha}_{k}=\mu^{m-k\left(f_{j}\right)}\left[\mu^{n_{j} r_{j}} \hat{\alpha}_{k\left(f_{j-1}\right)}+\sum_{t \in T} \hat{\alpha}_{k\left(f_{j}-t\right)} \mu^{t n_{j}}+\hat{\alpha}_{k\left(f_{j}\right)}\right]=0,
$$

where the expression in brackets is a polynomial in $\mu^{n_{j}}$. Now, we recall from Theorem 3.1 that for each $l=f_{j}-t$ with $t \in T$, the corresponding $\hat{\alpha}_{k(l)}$ is (up to the sign) the sum of all principal minors of $\Phi_{j}$ of dimension $l$ containing $\Phi_{j-1}$, which is precisely the way the coefficients of the powers of $\xi$ are obtained in $\operatorname{det}\left(\Phi_{j}-\xi E_{j}\right)$. This implies that we get the nonzero solutions of (3.3) by solving $\operatorname{det}\left(\Phi_{j}-\mu^{n_{j}} E_{j}\right)=0$.

Now suppose that $\Phi_{j}$ is singular. Then the corresponding point $\mathcal{P}_{j}$ no longer belongs to the diagram, implying the loss of some of the expansions (2.4) or, equivalently, the loss of part of the segment $S_{j}$. The part of $S_{j}$ that actually remains depends upon the nullity of $\Phi_{j}$. If $\operatorname{rank} \Phi_{j}=f_{j}-\beta$, there are $\beta$ rows or columns of $\Phi_{j}$ that depend linearly on the remaining ones. This means, on one hand, that no point $\mathcal{Q}_{t} \equiv\left(k\left(f_{j}-t\right), f_{j}-t\right)$ appears in the diagram for $1 \leq t<\beta$ and, on the other hand, that $\mathcal{Q}_{\beta}$ does appear (each principal minor of $\Phi_{j}$ of dimension $f_{j}-\beta$ either vanishes or takes a common nonzero value, since only $f_{j}-\beta$ columns of $\Phi_{j}$ are linearly independent). We conclude that the part of $S_{j}$ remaining in the Newton diagram is the segment connecting $\mathcal{P}_{j-1}$ to $\mathcal{Q}_{\beta}=\left(k\left(f_{j}-\beta\right), f_{j}-\beta\right)$ (see Fig. 3.2). This accounts for $\left(r_{j}-\beta\right) n_{j}$ expansions (2.4), whose leading coefficients are,

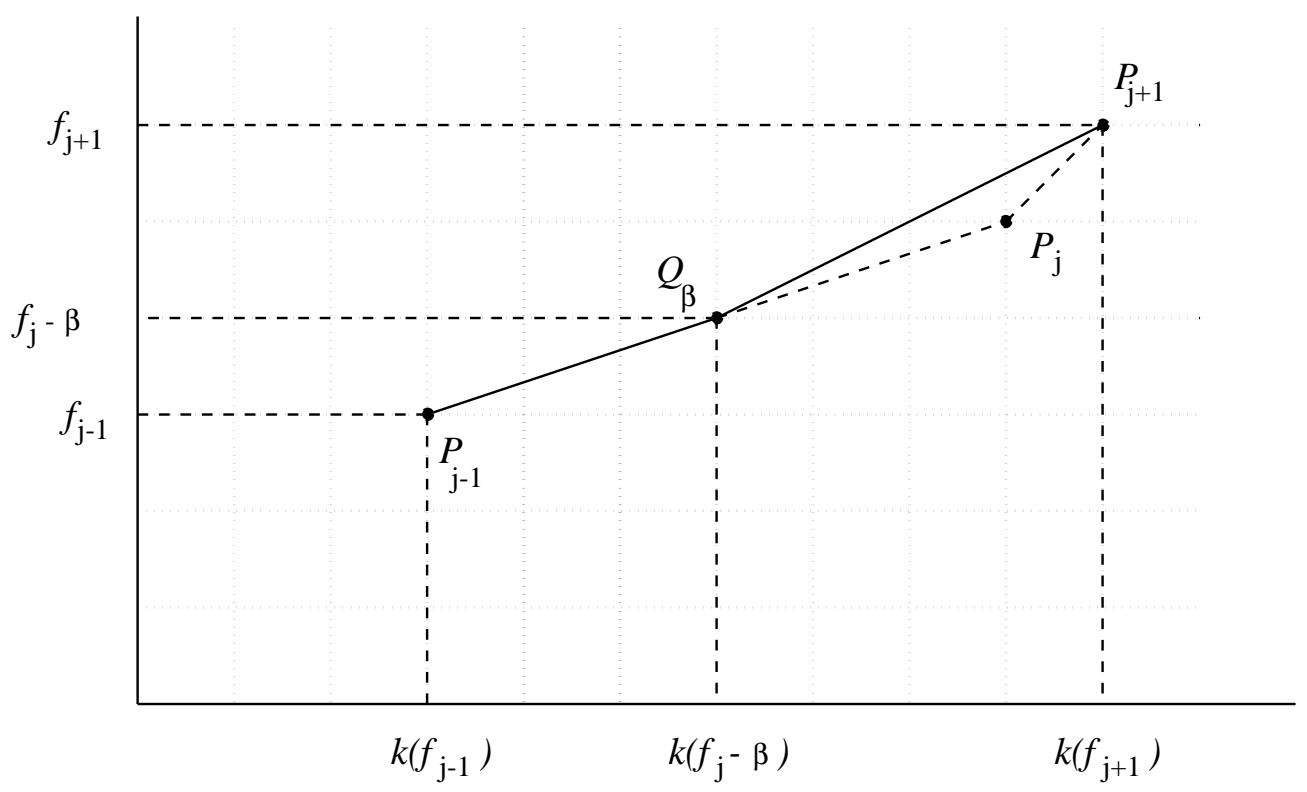

FIG. 3.2. The Newton diagram is shown as a solid line and the envelope as a dashed line.

reasoning as above, the $n_{j}$ th roots of the $r_{j}-\beta$ nonzero solutions of equation (2.5). As for the $\beta n_{j}$ remaining eigenvalues, they correspond to segments whose slope is strictly larger than $1 / n_{j}$. Hence, the remaining expansions (2.4) are still valid since they correspond to the $\beta$ null solutions of equation (2.5). 
A further consequence of this Newton diagram approach is that the hypotheses of Theorem 2.2 can be weakened in the sense that, if $j$ is such that all roots of (2.5) are distinct, we only need $\Phi_{1}, \ldots, \Phi_{j}$ to be nonsingular to guarantee the simplicity of the $r_{j} n_{j}$ eigenvalues $\lambda_{j}^{k l}(\varepsilon)$ : the slope of any segment of the diagram lying to the right of $\mathcal{P}_{j}$ is strictly larger than $1 / n_{j}$ regardless of the singularity of $\Phi_{s}, s=j+1, \ldots, q$. Hence, no eigenvalue corresponding to a Jordan block of size less than $n_{j}$ can coincide with any $\lambda_{j}^{k l}(\varepsilon)$ if $\varepsilon$ is small enough.

Apart from recovering the results of section 2, the approach through the Newton diagram is quite helpful in getting a better understanding of the nongeneric case when some $\Phi_{j}$ is singular. The fact that in this case $\mathcal{P}_{j}$ does not belong to the Newton diagram implies that neither the complete segment $S_{j}$ nor the complete segment $S_{j+1}$ appears on the Newton diagram. This indicates some kind of interaction between the eigenvalues associated with blocks of size $n_{j}$ and those associated with blocks of size $n_{j+1}$. We may, in fact, use the Newton diagram as a tool to quantify this interaction, actually finding both leading exponents and coefficients of the missing eigenvalue expansions in simple situations. The range of possibilities is easily visualized with the aid of the Newton envelope. For example, if $\left(h_{1}, k_{1}\right)$ and $\left(h_{2}, k_{2}\right)$ are two points that are known to lie on both the Newton diagram and the Newton envelope, then the segment of the Newton diagram between $h_{1}$ and $h_{2}$ must necessarily lie between the chord connecting $\left(h_{1}, k_{1}\right)$ to $\left(h_{2}, k_{2}\right)$ and the Newton envelope. Thus, to determine the Newton diagram one need only focus on the integer lattice points trapped between this chord and the Newton envelope. As an illustration of the power of this observation in the nongeneric case, we provide the following corollary. In this corollary, we identify a case in which no integer lattice points can lie between the chord and the Newton envelope.

Corollary 3.3. Let $0 \leq \beta \leq r_{j}$ and $0 \leq \alpha \leq r_{j+1}$. Suppose that $\mathcal{Q}_{\beta}^{j}=$ $\left(k\left(f_{j}-\beta\right), f_{j}-\beta\right)$ and $\hat{\mathcal{Q}}_{\alpha}^{j}=\left(k\left(f_{j}+\alpha\right), f_{j}+\alpha\right)$ are two points lying on the Newton diagram, while the points $\mathcal{Q}_{s}^{j}$ for $s=\beta-1, \ldots, 1, \mathcal{P}_{j}$ and $\hat{\mathcal{Q}}_{t}^{j}$ for $t=1,2, \ldots, \alpha-1$ do not lie on the Newton diagram. Set $p=\beta n_{j}+\alpha n_{j+1}$ and $\sigma=\frac{\beta+\alpha}{p}$. If

$$
\left(\sigma n_{j}-1\right) \beta \leq \min (\sigma, 1-\sigma),
$$

then there are $p$ eigenvalues of $A+\varepsilon B$ of the form

$$
\lambda^{l}(\varepsilon)=\lambda+\eta^{1 / p} \varepsilon^{\sigma}+o\left(\varepsilon^{\sigma}\right), \quad l=1,2, \ldots, p,
$$

where $\eta \neq 0$. Moreover, if either

(i) the inequality in (3.4) is strict or

then

(ii) $\left(\sigma n_{j}-1\right) \beta<\sigma$ and $\alpha=n_{j+1}=1$,

$$
\eta=-\frac{\hat{\alpha}_{k\left(f_{j}+\alpha\right)}}{\hat{\alpha}_{k\left(f_{j}-\beta\right)}} .
$$

Proof. As noted above, the Newton diagram must lie between the chord connecting $\mathcal{Q}_{\beta}^{j}$ to $\hat{\mathcal{Q}}_{\alpha}^{j}$ and the Newton envelope on the interval $\left[k\left(f_{j}-\beta\right), k\left(f_{j}+\alpha\right)\right]$. Furthermore, since none of the points $\mathcal{Q}_{s}^{j}$ for $s=\beta-1, \ldots, 1, \mathcal{P}_{j}$ and $\hat{\mathcal{Q}}_{t}^{j}$ for $t=1,2, \ldots, \alpha-1$ lie on the Newton diagram, the Newton envelope and diagram coincide only at the end points of this interval. Thus, the expansions (3.5) will be valid if we can show that there are no integer lattice points strictly between the chord and the Newton 
diagram on the interval $\left[k\left(f_{j}-\beta\right), k\left(f_{j}+\alpha\right)\right]$. To do this we need only show that the lattice points $\left(k\left(f_{j}\right)-1, f_{j}\right)$ and $\left(k\left(f_{j}\right)+1, f_{j}+1\right)$ lie on or above the chord. The condition that $\left(k\left(f_{j}\right)-1, f_{j}\right)$ lies on or above the chord yields the inequality $\left(\sigma n_{j}-1\right) \beta \leq \sigma$, while the condition that $\left(k\left(f_{j}\right)+1, f_{j}+1\right)$ lies on or above the chord yields the inequality $\left(\sigma n_{j}-1\right) \beta \leq 1-\sigma$. Note that this second condition is no longer needed if $\alpha=n_{j+1}=1$, since in this case $\left(k\left(f_{j}\right)+1, f_{j}+1\right)$ coincides with $\hat{\mathcal{Q}}_{\alpha}^{j}$. Thus, under either condition (i) or (ii), $\mathcal{Q}_{\beta}^{j}$ and $\hat{\mathcal{Q}}_{\alpha}^{j}$ are the only integer lattice points on the chord and so (3.6) follows from (3.3).

It is interesting to consider a few special cases of the above result. Note that if $\beta=0$, then $\alpha$ can take any of the values $0,1, \ldots, r_{j+1}$, and if $\alpha=0$, then $\beta$ can take any of the values $0,1, \ldots, r_{j}$. The case $\beta=0$ reaffirms the third remark at the end of section 2 , while the case $\alpha=0$ illustrates that expansions with power $1 / n_{j}$ are possible even if $\Phi_{j-1}$ is singular. Finally, if one is given a fixed value for either $\alpha$ or $\beta$, then simple bounds on the other value are easily obtained from (3.4). For example, if $\alpha=1$, then the restriction (3.4) yields the inequality $\beta\left(n_{j}-n_{j+1}-1\right) \leq 1$. That is, if $n_{j}=n_{j+1}+1$, then $\beta$ can take on any of the values $0,1, \ldots, r_{j}$; if $n_{j}=n_{j+1}+2$, then $\beta$ can take only the values 0 and 1 ; and if $n_{j}>n_{j+1}+2$, then $\beta$ must be zero.

Corollary 3.3 also explains the exponent appearing in the eigenvalues of Wilkinson's example (1.1). In that case, the point $\mathcal{P}_{1}=(3,1)$ does not lie on the Newton diagram since $\Phi_{1}=0$. On the other hand, $\mathcal{P}_{2}=(5,2)$ does appear, due to the nonsingularity of

$$
\Phi_{2}=\left[\begin{array}{ll}
0 & 1 \\
1 & 0
\end{array}\right]
$$

Thus, an application of Corollary 3.3 with $j=1, \alpha=\beta=1$ shows that there are $p=5$ eigenvalues of order $\sigma=2 / 5$.

The situation becomes more complicated with the introduction of more integer lattice points between the chord and the Newton envelope, since more possibilities for the Newton diagram arise. But, in many cases, these possibilities can be delineated by considering certain key lattice points as was done in the proof of the above corollary. Indeed, this approach can provide a fairly complete picture in many particular cases. Let us conclude by applying the ideas of this section to some specific examples.

Example 1. We first turn to our example in the preceding section of a $3 \times 3$ matrix with $q=2, n_{1}=2, n_{2}=1, r_{1}=r_{2}=1$. The expansions we obtained in the previous section correspond to a Newton diagram with two segments: $S_{1}$ connecting $(0,0)$ with $\mathcal{P}_{1}=(2,1)$ and $S_{2}$ joining $\mathcal{P}_{1}$ and $\mathcal{P}_{2}=(3,2)$. Note that this is precisely the Newton envelope corresponding to the given Jordan structure (see Fig. 3.3(a)). Now suppose that $\Phi_{1}=0$ with $\operatorname{det} \Phi_{2} \neq 0$. This means that $\mathcal{P}_{1}$ no longer is plotted, so the diagram consists of a single segment of slope $2 / 3$ joining $(0,0)$ with $\mathcal{P}_{2}$ (see Fig. 3.3(b)). If both $\Phi_{1}$ and $\Phi_{2}$ are singular, we obtain one single segment of slope 1 as long as $B$ is nonsingular.

Example 2. We consider a $5 \times 5$ matrix with one zero eigenvalue and $q=2, n_{1}=$ $2, n_{2}=1, r_{1}=2, r_{2}=1$. We assume, for the sake of simplicity, that $A$ is already in Jordan form, i.e., 


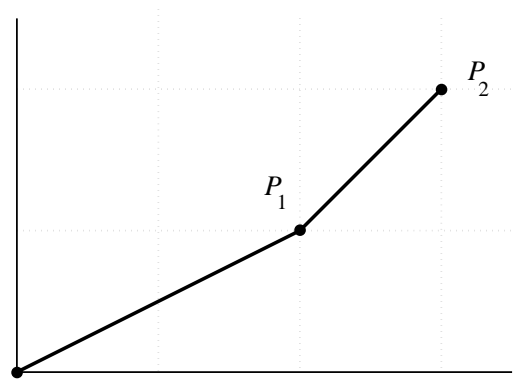

(a)

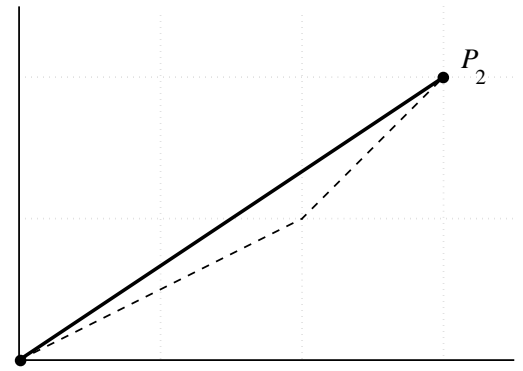

(b)

FIG. 3.3. Newton diagrams corresponding to Example 1. In (a) the Newton diagram and envelope coincide. In (b) the diagram is shown as a solid line and the envelope as a dashed line.

$$
A=\left[\begin{array}{llll|l}
0 & 1 & & & \\
& 0 & & & \\
& & 0 & 1 & \\
& & & 0 & \\
& & & & 0
\end{array}\right] .
$$

If $B=\left(b_{i j}\right)_{i, j=1}^{5}$, then

$$
\Phi_{1}=\left[\begin{array}{ll}
b_{21} & b_{23} \\
b_{41} & b_{43}
\end{array}\right], \quad \Phi_{2}=\left[\begin{array}{lll}
b_{21} & b_{23} & b_{25} \\
b_{41} & b_{43} & b_{45} \\
b_{51} & b_{53} & b_{55}
\end{array}\right] .
$$

We consider the different possibilities.

(i) Suppose $\operatorname{det} \Phi_{1} \neq 0$, so that $\mathcal{P}_{1}=(4,2)$ appears in the diagram. Then the perturbed matrix has four eigenvalues

$$
\lambda_{1}^{k l}(\varepsilon)=\left(\xi_{1}^{k}\right)^{1 / 2} \varepsilon^{1 / 2}+o\left(\varepsilon^{1 / 2}\right), \quad k, l=1,2,
$$

where $\xi_{1}^{1}, \xi_{1}^{2}$ are the eigenvalues of $\Phi_{1}$.

- If, additionally, $\operatorname{det} \Phi_{2} \neq 0$, so that $\mathcal{P}_{2}=(5,3)$ also appears in the diagram, then the fifth eigenvalue of $A+\varepsilon B$ is $\lambda_{2}^{11}(\varepsilon)=\xi_{2} \varepsilon+o(\varepsilon)$ for

$$
\xi_{2}=b_{55}-\left[\begin{array}{ll}
b_{51} & b_{53}
\end{array}\right] \Phi_{1}^{-1}\left[\begin{array}{l}
b_{25} \\
b_{45}
\end{array}\right] .
$$

In this case, the Newton diagram and envelope coincide (see Fig. 3.4 (a)).

- If, on the other hand, $\Phi_{2}$ is singular, $\mathcal{P}_{2}$ does not appear in the diagram. In this case, the order of the fifth eigenvalue is at least $O\left(\varepsilon^{2}\right)$, corresponding to a segment joining $\mathcal{P}_{1}=(4,2)$ with $(5,4)$. However, higher slopes might appear in some cases.

(ii) Suppose now that $\Phi_{1}$ is singular. Then the point $\mathcal{P}_{1}$ no longer appears in the Newton diagram, and to determine the order of the perturbations we need to know whether or not the coefficient

$$
\delta=-\operatorname{tr} \Phi_{1}=-b_{21}-b_{43}
$$




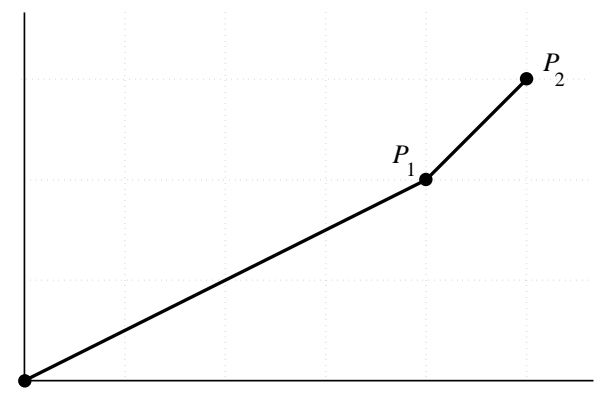

(a)

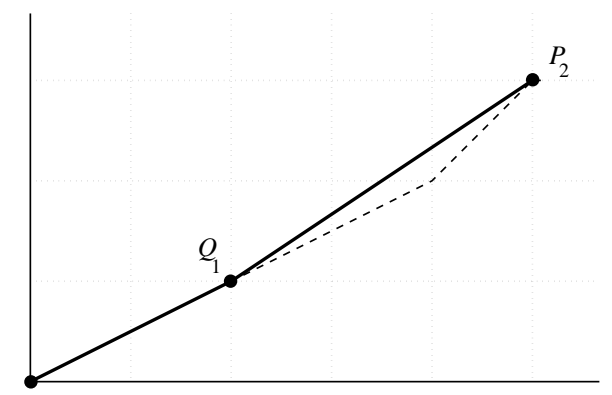

(b)

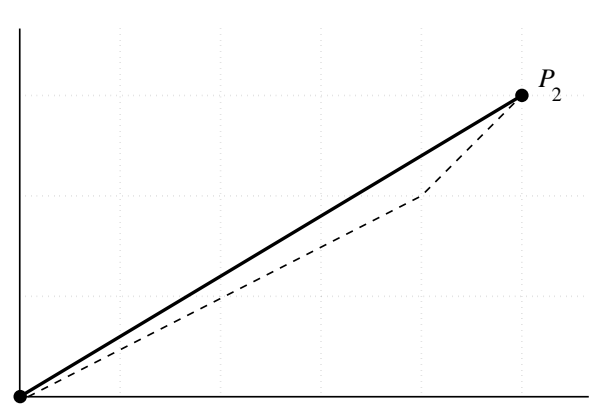

(c)

FIG. 3.4. Newton diagrams corresponding to Example 2. In (a) the Newton diagram and envelope coincide. In (b) and (c) the diagram is shown as a solid line and the envelope as a dashed line.

of $\varepsilon$ in $\alpha_{k(1)}$ is different from zero, i.e., whether or not $\mathcal{Q}_{1}=(2,1)$ is among the points plotted in the Newton diagram.

- If $\delta \neq 0$, then $a_{k(1)}=1$ and there is a segment of slope $1 / 2$ in the Newton diagram connecting $(0,0)$ and $\mathcal{Q}_{1}$. If, additionally, det $\Phi_{2} \neq 0$, there is a second segment of slope $2 / 3$ between $\mathcal{Q}_{1}$ and $\mathcal{P}_{2}=(5,3)$ (see Fig. $3.4(\mathrm{~b})$ ). Hence, two of the eigenvalues are

$$
\lambda_{1}^{l}(\varepsilon)=\delta^{1 / 2} \varepsilon^{1 / 2}+o\left(\varepsilon^{1 / 2}\right)
$$

since $\delta=\hat{\alpha}_{k(1)}$ is the unique nonzero eigenvalue of $\Phi_{1}$. The other three eigenvalues of $A+\varepsilon B$ may be expanded as

$$
\lambda_{2}^{l}(\varepsilon)=\eta^{1 / 3} \varepsilon^{2 / 3}+o\left(\varepsilon^{2 / 3}\right) \quad \text { for } \eta=\frac{\operatorname{det} \Phi_{2}}{\delta}
$$

applying Corollary 3.3 (ii) (in this case $\alpha=n_{2}=1$ and $\left(\sigma n_{1}-1\right) \beta=1 / 3<2 / 3=\sigma$ ).

- If $\delta=0$, then $a_{k(1)}>1$ and $\mathcal{Q}_{1}$ does not appear in the diagram. In the case when $\operatorname{det} \Phi_{2} \neq 0$, the Newton diagram consists of a single segment of slope $3 / 5$ connecting the origin with $\mathcal{P}_{2}$ (see Fig. 3.4(c)). The five eigenvalues are of the form

$$
\left(\operatorname{det} \Phi_{2}\right)^{1 / 5} \varepsilon^{3 / 5}+o\left(\varepsilon^{3 / 5}\right) .
$$

Finally, if both $\delta$ and $\operatorname{det} \Phi_{2}$ are zero, the actual slopes of the Newton diagram depend on the vanishing of the four-dimensional minors of $B$. 
4. Spectral condition numbers. The results of section 2 lead immediately to the proposition of a new notion of condition number for multiple eigenvalues.

Definition 4.1. Define the Hölder condition number of the eigenvalue $\lambda$ by

$$
\operatorname{cond}(\lambda)=\left(n_{1}, \alpha\right)
$$

where, as before, $n_{1}$ is the dimension of the largest Jordan block associated with $\lambda$ and

$$
\alpha=\max _{\|B\| \leq 1} \operatorname{spr}\left(Y_{1} B X_{1}\right),
$$

where spr denotes the spectral radius and the $r_{1}$ columns of $X_{1}$ (rows of $Y_{1}$ ) are independent right (left) eigenvectors of $\lambda$, each corresponding to a Jordan chain of greatest length $n_{1}$ as defined in section 2 .

The motivation for this definition is that $1 / n_{1}$ is the smallest possible power of $\varepsilon$ in the expansion of the eigenvalues of any perturbation $A+\varepsilon B$, while $\alpha^{1 / n_{1}}$ is the largest possible magnitude of the coefficient of $\varepsilon^{1 / n_{1}}$ in such expansions. Clearly, it follows from Theorem 2.1 that for all $c>1$, the eigenvalues $\lambda^{\prime}$ of $A+\varepsilon B$ converging to $\lambda$ as $\varepsilon \downarrow 0$ satisfy

$$
\left|\lambda^{\prime}-\lambda\right| \leq c \alpha^{1 / n_{1}} \varepsilon^{1 / n_{1}}
$$

for all sufficiently small positive $\varepsilon$. In fact, this bound is sharp in the sense that given $A$, there exists a perturbation $B$ such that for all $c<1$, (4.1) holds with the inequality reversed for some perturbed eigenvalue $\lambda^{\prime}$ when $\varepsilon$ is sufficiently small.

Note that the definition depends on the choice of matrix norm $\|\cdot\|$. We shall restrict our attention to unitarily invariant norms [6, p. 308].

THEOREM 4.2. If the condition number $\operatorname{cond}(\lambda)=\left(n_{1}, \alpha\right)$ is defined by any unitarily invariant matrix norm $\|\cdot\|$, then

$$
\alpha=\left\|X_{1} Y_{1}\right\|_{2} .
$$

Proof. One has

$$
\begin{aligned}
\max _{\|B\| \leq 1} \operatorname{spr}\left(Y_{1} B X_{1}\right) & =\max _{\|B\| \leq 1} \operatorname{spr}\left(B X_{1} Y_{1}\right) \\
& \leq \max _{\|B\| \leq 1}\left\|B X_{1} Y_{1}\right\|_{2} \\
& \leq\left\|X_{1} Y_{1}\right\|_{2},
\end{aligned}
$$

where the final inequality follows because $\|B\|_{2} \leq\|B\|$ for any unitarily invariant norm. To see that equality holds, note the following. Let the scalar $\sigma_{1}$, the row vector $u_{1}$, and the column vector $v_{1}$ be, respectively, the largest singular value and the corresponding left and right singular vectors of $X_{1} Y_{1}$, so that $\left\|u_{1}\right\|=\left\|v_{1}\right\|=1$ and $u_{1} X_{1} Y_{1} v_{1}=\sigma_{1}=\left\|X_{1} Y_{1}\right\|_{2}$. Setting $B=v_{1} u_{1}$ gives $\operatorname{spr}\left(B X_{1} Y_{1}\right)=\operatorname{spr}\left(u_{1} X_{1} Y_{1} v_{1}\right)=$ $\sigma_{1}$.

\section{Remarks.}

1. In the case in which $\lambda$ is simple, we have $n_{1}=r_{1}=1$, so $\operatorname{cond}(\lambda)=(1, \alpha)$, where $\alpha=\|x y\|=\|x\|\|y\|$, with the column vector $x$ and the row vector $y$, respectively, right and left eigenvectors for $\lambda$, normalized so that $y x=1$. Without loss of generality, one can take $\|x\|=1$, so $\alpha=\|y\|$. Thus the condition number reduces to the standard definition [4, p. 152]; see also [5, p. 202], where the normalization used is $\|x\|=\|y\|=1$, giving the definition $1 /(y x)$ for the condition number. 
2. In the case in which $\lambda$ is nonderogatory, we have $r_{1}=1$, so $\operatorname{cond}(\lambda)=\left(n_{1}, \alpha\right)$, where $\alpha=\|x y\|=\|x\|\|y\|$, with $x$ and $y$, respectively, right and left eigenvectors. However, when $n_{1}>1$, we have $y x=0$ (directly from the Jordan form). The normalization in this case is $Q_{1}^{1} P_{1}^{1}=I$, where the columns of $P_{1}^{1}$ (rows of $Q_{1}^{1}$ ) are right (left) Jordan chains for $\lambda, x$ being the first column of $P_{1}^{1}$ and $y$ the last row of $Q_{1}^{1}$. If $A$ is in Jordan form, then $P_{1}^{1}=Q_{1}^{1}=I$, so $\operatorname{cond}(\lambda)=\left(n_{1}, 1\right)$.

For example, take

$$
A=\left[\begin{array}{cc}
\delta & 1 \\
0 & -\delta
\end{array}\right]
$$

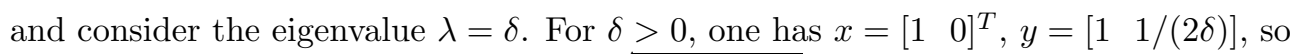
$\operatorname{cond}(\delta)=(1, \alpha)$ with $\alpha=\|x\|\|y\|=\sqrt{ } 1+1 /\left(4 \delta^{2}\right)$. Since the eigenvalue is simple, this condition number coincides with those given by [4] and [5]. As $\delta \downarrow 0$, the coefficient $\alpha$ in $\operatorname{cond}(\delta)$ diverges to $+\infty$. For $\delta=0$, the eigenvalue $\lambda=\delta$ has multiplicity two, so the definitions given in [4, p. 152] and [5] do not apply. In this case, $A$ is in Jordan form, so one has $x=\left[\begin{array}{ll}1 & 0\end{array}\right]^{T}, y=\left[\begin{array}{ll}0 & 1\end{array}\right]$, and cond $(0)=(2,1)$. Thus, although the condition number is not a continuous function of $\delta$ in the conventional sense, the divergence of $\alpha$ as $\delta \downarrow 0$ is reflected by the drop in the power $1 / n_{1}$ at the limit point.

Chatelin [4, p. 156] also introduced a closely related Hölder condition number in the more general context of clusters of eigenvalues. Let us restrict our attention to the case in which the cluster consists of one multiple eigenvalue $\lambda$ of multiplicity $n$. Chatelin defines a Hölder condition number $\operatorname{csp}(\lambda)=(n, \beta)$, with a coefficient $\beta$ which depends on the conditioning of the transformation reducing the matrix to Jordan form. Specifically, consider the matrices $P$ and $Q$ in (2.1), let $P$ have a "QR" factorization

$$
P=U R, \quad U^{*} U=I,
$$

and define $V=R Q$. Thus, the columns of $U$ form a unitary basis for the right invariant subspace for $\lambda$, while the rows of $V$ form a (nonunitary) basis for the left invariant subspace, satisfying the normalization condition $V U=R Q P R^{-1}=I$, since $Q P=I$ from (2.2). Then the Chatelin condition number $\operatorname{csp}(\lambda)=\left(n_{1}, \beta\right)$ has $\beta$ defined by

$$
\beta=\operatorname{cond}_{2}(R)\|V\|_{2},
$$

where $\operatorname{cond}_{2}(R)$ is the ordinary condition number $\|R\|_{2}\left\|R^{-1}\right\|_{2}$. (To see the equivalence with Chatelin's definition, note that $U^{*} A U=R J R^{-1}$, using $\widehat{Q} P=0$, again from (2.2).)

An important advantage of $\operatorname{cond}(\lambda)=\left(n_{1}, \alpha\right)$ over $\operatorname{csp}(\lambda)=\left(n_{1}, \beta\right)$ is that the coefficient $\alpha$ depends only on the left and right eigenvectors, not on the Jordan vectors. As a consequence, we obtain the following relation between both condition numbers.

LEMMA 4.3. If the condition number $\operatorname{cond}(\lambda)=\left(n_{1}, \alpha\right)$ is defined by any unitarily invariant matrix norm, then it is related to $\operatorname{csp}(\lambda)=\left(n_{1}, \beta\right)$ by

$$
\alpha \leq \beta .
$$


Proof. First, note that

$$
\alpha=\left\|X_{1} Y_{1}\right\|_{2} \leq\left\|X_{1}\right\|_{2}\left\|Y_{1}\right\|_{2} \leq\|P\|_{2}\|Q\|_{2}
$$

since the columns of $X_{1}$ (resp., rows of $Y_{1}$ ) are a subset of those of $P$ (resp., $Q$ ). Now, $P=U R$ with $U^{*} U=I$ and $Q=R^{-1} V$, so

$$
\alpha \leq\|P\|_{2}\|Q\|_{2}=\|R\|_{2}\left\|R^{-1} V\right\|_{2} \leq \operatorname{cond}_{2}(R)\|V\|_{2}=\beta .
$$

Consider, for example,

$$
A=\left[\begin{array}{ll}
0 & \delta \\
0 & 0
\end{array}\right]
$$

with a double eigenvalue $\lambda=0$. When $\delta>0$, the Jordan form of $A$ is given by

$$
\left[\begin{array}{ll}
0 & 1 \\
0 & 0
\end{array}\right]=P^{-1} A P, \quad P=\left[\begin{array}{cc}
1 & 0 \\
0 & 1 / \delta
\end{array}\right]
$$

with right eigenvector equal to the first column of $P$, i.e., $x=\left[\begin{array}{ll}1 & 0\end{array}\right]^{T}$, and left eigenvector equal to the second row of $P^{-1}, y=\left[\begin{array}{ll}0 & \delta\end{array}\right]$. Thus, for $\delta>0$, $\operatorname{cond}(0)=$ $(2, \alpha)$ with $\alpha=\|x\|\|y\|=\delta$. The Chatelin condition number is $\operatorname{csp}(0)=(2, \beta)$ with $\beta=\max \{\delta, 1 / \delta\}$, the condition number of $P$ in the 2-norm. As $\delta \downarrow 0$, the coefficient $\alpha$ in $\operatorname{cond}(\lambda)=(2, \alpha)$ converges to zero, as it should since at the limit point the power $1 / n_{1}$ increases to 1 , giving the perfect condition number $\operatorname{cond}(0)=(1,1)$. However, the coefficient $\beta$ in $\operatorname{csp}(0)=(2, \beta)$ diverges to $+\infty$, even though the condition number in the limiting case $\delta=0$ is also $\operatorname{csp}(0)=(1,1)$.

The condition number $\operatorname{cond}(\lambda)$ is trivially extended to clusters of eigenvalues by defining it to be the lexicographic maximum of the ordered pairs defining the condition number for each element of the cluster.

Acknowledgments. The third author thanks Prof. A. Seyranian for bringing [13] to his attention several years ago and for suggesting the dedication of this paper. The authors also thank an anonymous referee for a number of helpful suggestions.

\section{REFERENCES}

[1] H. Baumgärtel, Analytic Perturbation Theory for Matrices and Operators, Birkhäuser-Verlag, Basel, Switzerland, 1985.

[2] J. V. Burke And M. L. Overton, Stable perturbations of nonsymmetric matrices, Linear Algebra Appl., 171 (1992), pp. 249-273.

[3] J. V. Burke And M. L. Overton, Differential properties of the spectral abscissa and the spectral radius for analytic matrix-valued mappings, Nonlinear Anal., 23 (1994), pp. 467488.

[4] F. Chatelin, Eigenvalues of Matrices, John Wiley, New York, 1993.

[5] G. Golub and C. F. Van Loan, Matrix Computations, 1st ed., The Johns Hopkins University Press, Baltimore, MD, 1983.

[6] R. Horn and C. R. Johnson, Matrix Analysis, Cambridge University Press, Cambridge, UK, 1990.

[7] T. Kato, Perturbation Theory for Linear Operators, Springer-Verlag, Berlin, 1980.

[8] H. Langer and B. Najman, Remarks on the perturbation of analytic matrix functions III, Integral Equations Operator Theory, 15 (1992), pp. 796-806. 
[9] H. LANGER AND B. NAJMAN, Leading coefficients of the eigenvalues of perturbed analytic matrix functions, Integral Equations Operator Theory, 16 (1993), pp. 600-604.

[10] V. B. LidskiI, Perturbation theory of non-conjugate operators, U.S.S.R. Comput. Math. and Math. Phys., 1 (1965), pp. 73-85 (Zh. vychisl. Mat. mat. Fiz., 6 (1965), pp. 52-60).

[11] J. H. Maddocks AND M. L. Overton, Stability theory for dissipatively perturbed Hamiltonian systems, Comm. Pure Appl. Math., 48 (1995), pp. 583-610.

[12] M. M. Vainberg and V. A. Trenogin, Theory of Branching of Solutions of Non-linear Equations, P. Noordhoff, Leyden, 1974.

[13] M. I. VISHIK AND L. A. LyUSTERNIK, The solution of some perturbation problems for matrices and selfadjoint or non-selfadjoint differential equations I, Russian Math. Surveys, 15 (1960), pp. 1-74 (Uspekhi Mat. Nauk, 15 (1960), pp. 3-80).

[14] J. H. Wilkinson, The Algebraic Eigenvalue Problem, Clarendon, Oxford, UK, 1965. 